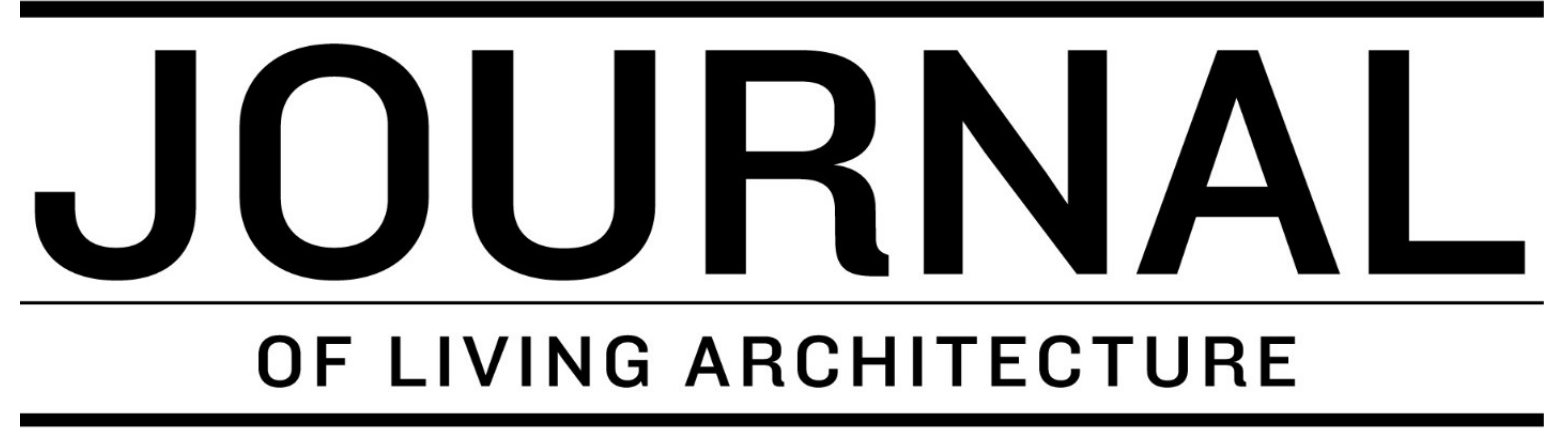

A GREEN ROOFS FOR HEALTHY CITIES PUBLICATION

\title{
Observations on the survival capacity of 118 plant taxa on a green roof in a semi-arid climate: 12 year update
}

\author{
Amy Schneider ${ }^{1}$, Michelle Landis ${ }^{2}$, Jennifer Bousselot ${ }^{2,}$, \\ ${ }^{1}$ Denver Botanic Gardens, 1007 York Street, Denver, CO 80206, \\ ${ }^{2}$ Department of Horticulture and Landscape Architecture, Colorado State University, Ft. Collins, CO 80523 \\ *corresponding author: Jennifer.Bousselot@,colostate.edu
}

\begin{abstract}
The Green Roof at Denver Botanic Gardens was built in the fall of 2007. Since installation, data on the green roof have been collected on 118 plant taxa 43 of which have survived for over 10 years. Plants were grouped based on their metabolic and growth type: succulents, creeping forbs, upright forbs, graminoid, and shrubs. Overall, shrubs and succulents have displayed the highest survival rates, showing the greatest potential for use on green roofs in semi-arid conditions. Graminoids survived about half the time and creeping and upright forbs had the lowest overall survival in semi-arid Colorado. Species survivability rates were calculated based on the number of plants of that species originally installed on the green roof.
\end{abstract}

Key words: Green Roof, Long-Term Species Evaluations, Semi-Arid

\section{INTRODUCTION}

Green roofs are constructed ecosystems installed on rooftops in cities to help mitigate the negative effects of urbanization and add space for biodiversity. Green roof environmental benefits include: urban heat island mitigation, stormwater runoff management, utilization as urban agriculture sites, restoration of urban biodiversity, building energy savings, and aesthetics (Getter and Rowe, 2006; Oberndorfer et al., 2007). Several US cities such as Denver, CO, 
Philadelphia, PA, Seattle, WA, New York, NY, Chicago, IL, (and more) recognize these benefits and have developed incentives to increase green roof installations.

Green roofs that contain a diversity of plant species and plant forms have been shown to outperform monocultures, providing greater ecosystem services (Lundholm \& Cadotte, 2015). Diverse plant communities with many plant forms, such as shrubs, grasses, and herbaceous perennials increase the total cooling effect that the green roof system can offer (Cao et al., 2019), lead to greater stormwater detention (Lundholm et al., 2010), and result in an increase in arthropod richness on the rooftop (Braaker et al., 2017).

Plant species diversity also benefits individual plants on a green roof. A range of plant forms that grow at different heights, causing shading, can influence overall plant success on a green roof (Aguiar et al., 2019). Plant species selection based on substrate depth and moisture retention of the substrate results in optimal survival rates (Graceson et al., 2014; Nektarios et al., 2011). Long-term diverse plant taxa evaluations on green roofs in North America have seldomly been published. Only one other long-term study in North America by Lubell et al. (2017), is found in literature.

The harsh environment on green roofs can cause many challenges for plants because of higher intensity of light, higher and lower air and substrate temperatures, relatively shallow substrate depth, wind gusts, low water availability, and more intense storm effects. Therefore, taxa planted must be selected for both survivability and enhanced benefits (Shafique et. al, 2018, Reyes et al., 2016). Sedum spp. are commonly used on green roofs because of their ability to efficiently utilize moisture and survive in dry locations. Green roofs that contain a diverse range of plant taxa have been shown to outperform monocultures, providing greater ecosystem services (Lundholm and Cadotte, 2015).

Plant provenance could play a role on plant survivability on green roofs, but more research must be conducted, especially in North America (Dvorak and Volder 2010). A ten-year study of native plant survival conducted in the Northeastern U.S. by Lubell et al (2017) found that approximately $50 \%$ of the initial plant taxa perished. In a three-year evaluation of native plants conducted in Michigan, $67 \%$ of native plant taxa had perished when no supplemental irrigation was added after establishment (Monterusso et al., 2005).

To date, 118 plant taxa have been trailed on the semi-arid Denver Botanic Gardens (DBG) green roof over the past 12 years. The DBG green roof, along the Front Range of Colorado, is used to demonstrate potential plant taxa for use on green roofs with similar environments and limited moisture. Choosing plant taxa suited to the climate, and especially the precipitation of an area, can change the overall air temperature cooling effects, reducing or enhancing the success of a green roof (Chagolla-Aranda et al., 2017). Plant taxa in this study were characterized by plant metabolic pathway and growth form, as in evaluations conducted by MacIvor and Lundholm (2011). The objective was to evaluate plant taxa in this observational study for long-term success as potential green roof taxa used in a semi-arid climate. Additionally, we discuss how plant taxa provenance may have influenced survivability. 


\section{MATERIALS AND METHODS}

Located along the Front Range of Denver, Colorado, USA in USDA plant hardiness zone 5 at an elevation of 1609 meters above sea level, the DBG Green Roof was installed on the south side of a one-story building, the Boettcher Memorial Tropical Conservatory. Established in the fall of 2007, this is one of Denver's earliest green roof installations (Figure 1).

The green roof is $195 \mathrm{~m}^{2}$ with substrate depth gradually increasing from $10 \mathrm{~cm}$ at the northwest edge to $45.7 \mathrm{~cm}$ near the southeast corner, which creates a small berm. This green roof is considered semi-intensive, which allows us to evaluate a range of plant taxa suited to variable substrate depths. We did not control for substrate depth or microclimates on this green roof as this is an observational study of survivability only. The substrate consists of $80 \%$ expanded shale and $20 \%$ compost/composted bark, by volume. During the initial planting in 2007 , mycorrhizae were added to the substrate, and in 2009 local compost was applied at the rate of approximately $6 \mathrm{~mm}$ on the surface. Since then, no supplemental nutrients have been applied.

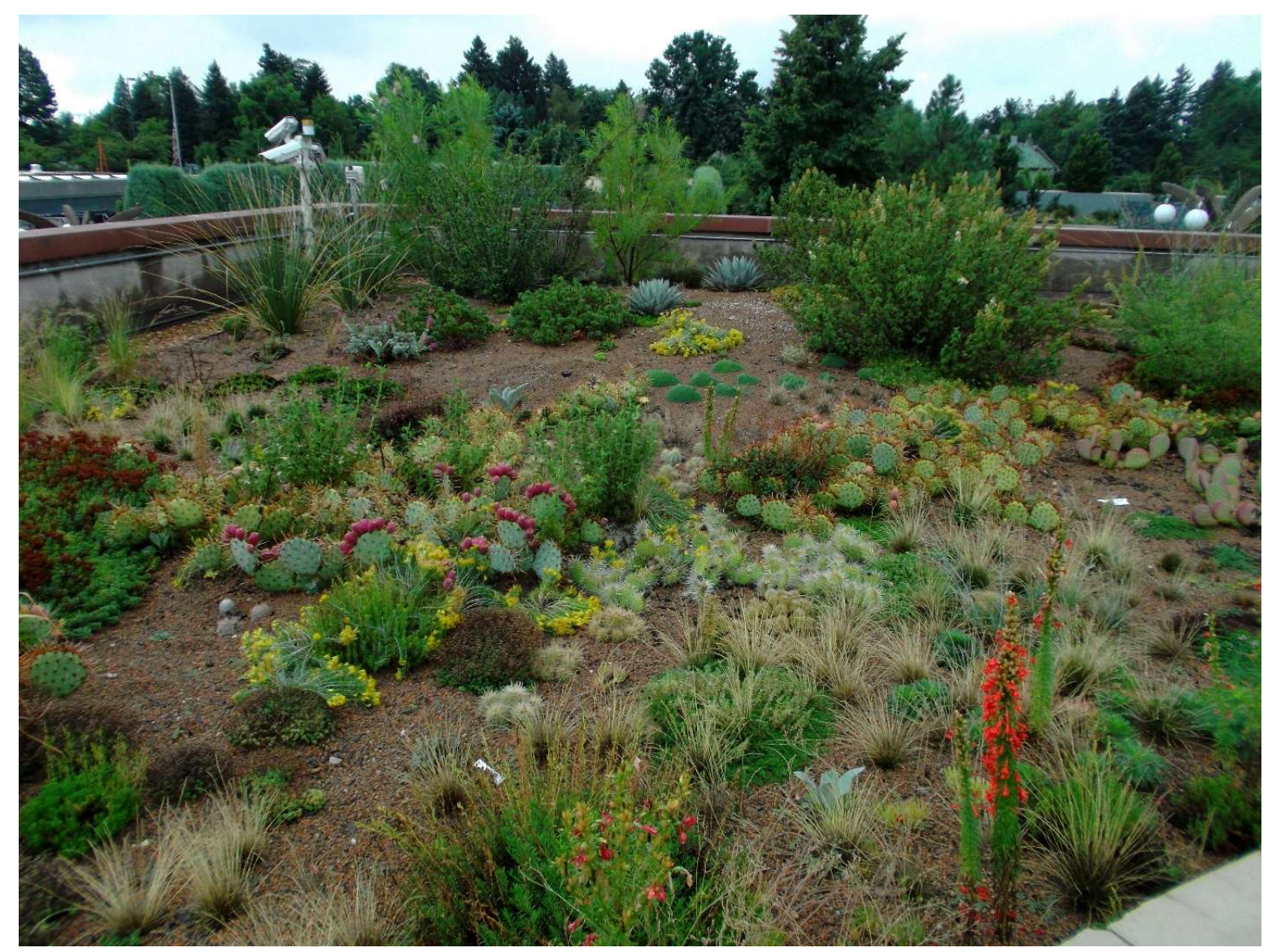

Figure 1. The Denver Botanic Gardens (DBG) green roof in September 2013. Photo by Amy Schneider.

\section{Environmental Conditions}

Microclimates are created by shrubs and the three-foot-high concrete parapet walls that surround the east, south and western edges of the space. In the winter, the roof heats up during the day due to reflection from the surrounding surfaces and drops to freezing temperatures 
overnight. The extreme seasonal fluctuations on the green roof, low moisture holding capacity of the substrate, and shallow depth of the substrate make survival for plants difficult.

The Colorado Front Range is a temperate and semi-arid region averaging from $18^{\circ} \mathrm{C}$ in warmer months to $2^{\circ} \mathrm{C}$ in the cooler months with an average annual precipitation rate of about $350 \mathrm{~mm}$. Precipitation at DBG was collected at the southwest corner of the gardens (Table 1). Due to low precipitation and extreme summertime heat, irrigation was installed on the green roof at planting time. Half of the green roof was irrigated by an underground drip and the other half by popup sprinkler heads. For initial plant establishment, between 2007-2009, approximately 100$150 \mathrm{~mm}$ of water through irrigation was provided per month throughout the growing season with some supplemental hand watering. From 2011 on, the irrigation system is always activated in July with an annual irrigation rate of $200 \mathrm{~mm}$ or less. In 2009, 2011, 2014, and 2015, annual precipitation at DBG was exceptionally high, over $500 \mathrm{~mm}$. A total of $1020 \mathrm{~mm}$ of supplemental irrigation has been applied to the green roof between the years of 2010-2019.

Table 1. Total monthly precipitation (in $\mathrm{mm}$ ) and first/last snow at the Denver Botanic Gardens (DBG) from 2007 to 2019 (CoCoRaHS, n.d; accessed January 2020).

\begin{tabular}{|c|c|c|c|c|c|c|c|c|c|c|c|c|c|}
\hline Month & 2007 & 2008 & 2009 & 2010 & 2011 & 2012 & 2013 & 2014 & 2015 & 2016 & 2017 & 2018 & 2019 \\
\hline Jan & 4.3 & 11.4 & 12.2 & 4.1 & 13.7 & 11.7 & 7.4 & 27.2 & 10.7 & 8.9 & 22.6 & 10.7 & 45.5 \\
\hline Feb & 0.8 & 14.7 & 3.8 & 19.1 & 27.2 & 50.5 & 26.4 & 8.4 & 60.5 & 23.6 & 4.1 & 10.4 & 23.4 \\
\hline Mar & 33.3 & 20.6 & 33.3 & 47.5 & 8.6 & Trace & 40.9 & 27.7 & 22.1 & 63.5 & 21.3 & 50.5 & 52.3 \\
\hline Apr & 70.1 & 29.5 & 114.0 & 56.1 & 22.1 & 31.8 & 69.6 & 38.9 & 51.6 & 10.4 & 61.7 & 32.8 & 21.3 \\
\hline May & 97.5 & 57.2 & 61.0 & 55.9 & 111.8 & 37.1 & 47.0 & 102.6 & 161.8 & 72.1 & 61.2 & 67.6 & 99.6 \\
\hline Jun & 12.7 & 17.0 & 85.1 & 52.3 & 43.4 & 10.4 & 13.5 & 30.5 & 219.2 & 49.3 & 28.7 & 28.2 & 30.7 \\
\hline Jul & 26.2 & 5.8 & 91.2 & 30.5 & 168.9 & 57.9 & 70.9 & 118.6 & 40.6 & 17.8 & 29.7 & 59.7 & 44.7 \\
\hline Aug & 55.6 & 108.7 & 25.9 & 11.2 & 17.8 & 9.9 & 42.7 & 38.4 & 23.6 & 39.4 & 22.6 & 24.6 & 32.3 \\
\hline Sep & 18.0 & 37.6 & 21.3 & 3.0 & 36.1 & 68.1 & 139.2 & 52.6 & 7.1 & 1.3 & 38.6 & 43.9 & 20.0 \\
\hline Oct & 30.2 & 18.3 & 67.8 & 14.2 & 47.2 & 31.0 & 17.5 & 27.9 & 54.4 & 2.5 & 32.0 & 29.2 & 7.4 \\
\hline Nov & 5.6 & 5.6 & 26.9 & 15.0 & 19.6 & 9.1 & 4.8 & 16.8 & 59.4 & 17.8 & 6.6 & 9.1 & \\
\hline Dec & 42.4 & 13.2 & 14.5 & 7.4 & 43.4 & 5.8 & 10.2 & 18.5 & 27.9 & 20.1 & 3.0 & 2.5 & \\
\hline Total & 396.7 & 339.6 & 557.0 & 316.2 & 559.8 & 323.3 & 490.0 & 508.0 & 738.9 & 326.6 & 332.2 & 369.3 & 377.2 \\
\hline $\begin{array}{l}\text { Last } \\
\text { Snow }\end{array}$ & $4 / 14$ & $5 / 14$ & $4 / 25$ & $5 / 12$ & $4 / 23$ & $4 / 3$ & $5 / 2$ & $5 / 12$ & $5 / 10$ & $4 / 29$ & $5 / 18$ & $4 / 24$ & $5 / 21$ \\
\hline $\begin{array}{l}\text { First } \\
\text { Snow }\end{array}$ & $10 / 22$ & $11 / 13$ & $10 / 10$ & $11 / 10$ & $10 / 26$ & $10 / 5$ & $10 / 4$ & $11 / 11$ & $11 / 5$ & $11 / 17$ & $10 / 9$ & $10 / 31$ & $10 / 10$ \\
\hline
\end{tabular}

\section{Plants}

During the past 12 growing seasons, from 2007-2019, 118 plant taxa have been trialed on the green roof. Many of the taxa were obtained from onsite propagation at DBG, grown from seed or cuttings, and a few presented spontaneously. Most of the 118 plant taxa trialed are generally available in the local green industry.

Planting dates for the plant taxa varied between 2007-2019 (Table 2, 3, and 4). Plants were installed on the green roof for aesthetic design purposes and were therefore massed. As plants perished they were either replanted or replaced by a different plant taxa. In 2007, during the installation, 53 plant taxa were planted at the beginning of the trial, ten new taxa were planted in 2008, five planted in 2009, 16 more were added in 2010, 20 in 2011, seven in 2012, two in 
2014, one in 2016, and 4 new plant taxa were planted in 2019 (including the spontaneous Muhlenbergia sp.). No new plants were planted in 2013, 2015, 2017, or 2018.

For this evaluation, plants were categorized by plant growth form characteristics, as in a study completed in Halifax, Canada (MacIvor and Lundholm, 2011). The plant groups represented in this study include: succulents, creeping forbs (which in this evaluation only represents nonsucculents), upright forbs (represents all non-carpet growing forbs), graminoids, and woody shrubs. The creeping forbs were also subcategorized into bun forming forbs because they do not spread as rapidly as the other creeping forbs. All plant taxa were monitored for 12 years and designated as 'perish' or 'survive'. The 'survive' category was then subdivided into 2 groups: plant taxa surviving over 10 years and plant taxa currently surviving under 10 years.

Plant taxa provenance information was included to determine if provenance could help explain survival rate in each plant category. In the succulent category, 15 of 22 planted taxa are native to North America, in the creeping forbs category, 15 of the total 47 are native, 13 of the 24 upright forbs, all 8 of the graminoids (excluding Muhlenbergia sp. and Andropogon sp. found in 2019), and of the woody shrubs, 12 of the 15 are native to North America.

Table 2. All taxa surviving more than 10 years by scientific name, common name, plant growth form, months surviving (two dates means replanting), and provenance.

\begin{tabular}{|c|c|c|c|c|}
\hline Scientific Name & Common Name & Form & $\begin{array}{l}\text { Months } \\
\text { since } \\
\text { planting } \\
\text { (year } \\
\text { planted) }\end{array}$ & $\begin{array}{l}\text { Provenance (Kew } \\
\text { science, n.d.) }\end{array}$ \\
\hline Acantholimon armenum & acantholimon, spike thrift & creeping forb, bun & $137(2008)$ & Asia minor \\
\hline Acantholimon litvinovii & acantholimon, spike thrift & creeping forb, bun & $137(2008)$ & $\begin{array}{l}\text { Central Asia } \\
\text { (Kirgizstan, } \\
\text { Uzbekistan) }\end{array}$ \\
\hline Agave parryi & Parry's agave & succulent & $137(2008)$ & $\begin{array}{l}\text { S.W. United States, N. } \\
\text { Mexico }\end{array}$ \\
\hline $\begin{array}{l}\text { Amorpha fruticosa } \\
\text { 'Nana' }\end{array}$ & dwarf false indigo & woody shrub & $125(2009)$ & S. United States* \\
\hline $\begin{array}{l}\text { Arctostaphylos 'Lauren's } \\
\text { Best' }\end{array}$ & Lauren's best manzanita & woody shrub & $143(2007)$ & W. North America* \\
\hline Arctostaphylos patula & greenleaf manzanita & woody shrub & $143(2007)$ & $\begin{array}{l}\text { W. United States, } \\
\text { N.W. Mexico }\end{array}$ \\
\hline $\begin{array}{l}\text { Arctostaphylos } \mathrm{x} \\
\text { coloradoensis 'Cascade' }\end{array}$ & cascade manzanita & woody shrub & $143(2007)$ & W. United States* \\
\hline Cercocarpus breviflorus & hairy mountain mahogany & woody shrub & $143(2007)$ & $\begin{array}{l}\text { S.W. United States } \\
\text { (AZ, NM, TX), N. } \\
\text { Mexico }\end{array}$ \\
\hline $\begin{array}{l}\text { Chamaebatiaria } \\
\text { millefolium }\end{array}$ & desert sweet & woody shrub & $143(2007)$ & W. United States \\
\hline Chilopsis linearis & desert willow & woody shrub & $143(2007)$ & $\begin{array}{l}\text { S.W. United States } \\
\text { (incl. Georgia) }\end{array}$ \\
\hline Dianthus anatolicus & Anatolian pink & creeping forb, bun & $137(2008)$ & Asia minor \\
\hline Draba hispanica & Spanish draba & creeping forb & $143(2007)$ & $\begin{array}{l}\text { N.W. Africa, S.W. } \\
\text { Europe (Spain) }\end{array}$ \\
\hline Echinocereus fendleri & Fendler's hedgehog cactus & succulent & 125 (2009) & S.W. United States \\
\hline
\end{tabular}




\begin{tabular}{|c|c|c|c|c|}
\hline $\begin{array}{l}\text { Echinocereus } \\
\text { triglochidiatus }\end{array}$ & kingcup cactus & succulent & $143(2007)$ & $\begin{array}{l}\text { S.W. United States } \\
\text { (AZ, NM, CO) }\end{array}$ \\
\hline $\begin{array}{l}\text { Echinocereus } \\
\text { triglochidiatus white } \\
\text { sands strain }\end{array}$ & $\begin{array}{l}\text { White sands kingcup } \\
\text { cactus }\end{array}$ & succulent & $143(2007)$ & S.W United States \\
\hline Echinocereus viridiflorus & nylon hedgehog cactus & succulent & $143(2007)$ & $\begin{array}{l}\text { C. United States, NE. } \\
\text { Mexico }\end{array}$ \\
\hline Echium amoenum & red feathers & upright forb & $143(2007)$ & $\begin{array}{l}\text { Middle East (Iran, } \\
\text { Transcaucasus) }\end{array}$ \\
\hline $\begin{array}{l}\text { Ericameria nauseosa } \\
\text { subsp. nauseosa var. } \\
\text { nauseosa }\end{array}$ & rubber rabbitbrush & woody shrub & $143(2007)$ & C. United States \\
\hline $\begin{array}{l}\text { Eriogonum umbellatum } \\
\text { var. aureum }\end{array}$ & sulphur flower buckwheat & creeping forb & $\begin{array}{l}143(2007), \\
137(2008)\end{array}$ & $\begin{array}{l}\text { W., N.W. United } \\
\text { States } \\
\text { (excl. WA, CA) }\end{array}$ \\
\hline Escobaria missouriensis & Missouri foxtail cactus & succulent & $143(2007)$ & $\begin{array}{l}\text { C., M.W. United } \\
\text { States }\end{array}$ \\
\hline Escobaria vivipara & spinystar & succulent & $143(2007)$ & $\begin{array}{l}\text { C., S.W. North } \\
\text { America }\end{array}$ \\
\hline $\begin{array}{l}\text { Euonymus nanus var. } \\
\text { turkestanicus }\end{array}$ & Turkistan burning bush & woody shrub & $143(2007)$ & $\begin{array}{l}\text { C. Asia } \\
\text { (Altai Mountains) }\end{array}$ \\
\hline Hesperaloe parviflora & $\begin{array}{l}\text { red yucca, hummingbird } \\
\text { yucca }\end{array}$ & succulent & $\begin{array}{l}143(2007), \\
137(2008)\end{array}$ & $\begin{array}{l}\text { S. United States (TX), } \\
\text { N.E. Mexico }\end{array}$ \\
\hline Heterotheca jonesii & Jones' false goldenaster & creeping forb & $143(2007)$ & $\begin{array}{l}\text { W. United States } \\
\text { (Utah) }\end{array}$ \\
\hline Iris germanica dwarf & dwarf Germanic iris & upright forb & $143(2007)$ & $\begin{array}{l}\text { S.E. Europe } \\
\text { (Yugoslavia) }\end{array}$ \\
\hline Maihuenia poeppigii & chupasangre & succulent & $137(2008)$ & $\begin{array}{l}\text { S. South America } \\
\text { (Argentina, Chile) }\end{array}$ \\
\hline Nolina microcarpa & beargrass & succulent & 143 (2007) & $\begin{array}{l}\text { S.W. United States } \\
\text { (AZ, NM), Mexico }\end{array}$ \\
\hline Opuntia aurea & golden pricklypear & succulent & $143(2007)$ & $\begin{array}{l}\text { W., S.W. United } \\
\text { States (AZ, UT) }\end{array}$ \\
\hline Opuntia phaeacantha & tulip pricklypear & succulent & $143(2007)$ & $\begin{array}{l}\text { W., S.W. United } \\
\text { States, N. Mexico }\end{array}$ \\
\hline Opuntia polyacantha & plains pricklypear & succulent & $143(2007)$ & $\begin{array}{l}\text { C., M.W. United } \\
\text { States, Canada }\end{array}$ \\
\hline Pediocactus simpsonii & mountain ball cactus & succulent & $143(2007)$ & $\begin{array}{l}\text { Central Western } \\
\text { United States }\end{array}$ \\
\hline Penstemon pinifolius & pineleaf penstemon & creeping forb & $125(2009)$ & $\begin{array}{l}\text { S.W. United States } \\
\text { (AZ, NM), Mexico }\end{array}$ \\
\hline $\begin{array}{l}\text { Phedimus kamtschaticus/ } \\
\text { Sedum kamtschaticum }\end{array}$ & Russian stone crop & succulent & $143(2007)$ & E., N.E. Asia \\
\hline Phemeranthus calycinus & largeflower flameflower & succulent & $\begin{array}{l}137(2008), \\
101(2011)\end{array}$ & $\begin{array}{l}\text { C., M.W., S. United } \\
\text { States, Mexico }\end{array}$ \\
\hline $\begin{array}{l}\text { Phlox bifida 'Betty } \\
\text { Blake' }\end{array}$ & cleft phlox & creeping forb & $143(2007)$ & $\begin{array}{l}\text { C. E. United States } \\
\text { and VT* }\end{array}$ \\
\hline Physaria bellii & front range twinpod & creeping forb & $143(2007)$ & $\begin{array}{l}\text { M.W. United States, } \\
\text { CO }\end{array}$ \\
\hline Salvia greggii & autumn sage & woody shrub & $137(2008)$ & $\begin{array}{l}\text { S. United States (TX), } \\
\text { NE. Mexico }\end{array}$ \\
\hline Salvia pachyphylla & mountain desert sage & upright forb & $143(2007)$ & $\begin{array}{l}\text { S.W. United States, } \\
\text { Mexico }\end{array}$ \\
\hline Sedum lanceolatum & spearleaf stonecrop & succulent & $143(2007)$ & $\begin{array}{l}\text { W., N.W. North } \\
\text { America }\end{array}$ \\
\hline Thelesperma ambiguum & Colorado greenthread & upright forb & $143(2007)$ & $\begin{array}{l}\text { S.W. United States } \\
\text { (TX and NM), N.E. } \\
\text { Mexico }\end{array}$ \\
\hline Veronica liwanensis & Turkish speedwell & creeping forb & $143(2007)$ & Asia minor \\
\hline
\end{tabular}




\begin{tabular}{|l|l|l|l|l|}
\hline & & & & $\begin{array}{l}\text { (Transcaucasus, } \\
\text { Turkey) }\end{array}$ \\
\hline Veronica pectinata & woolly speedwell & creeping forb & $143(2007)$ & $\begin{array}{l}\text { Asia minor (Bulgaria, } \\
\text { Turkey, Turkey-in- } \\
\text { Europe) }\end{array}$ \\
\hline Veronica thymoides & thyme-leaf speedwell & creeping forb & $143(2007)$ & Asia minor (Turkey) \\
\hline
\end{tabular}

Note: * includes plants that are cultivars

Table 3. All taxa surviving less than 10 years by scientific name, common name, plant growth form, months surviving, and provenance.

\begin{tabular}{|c|c|c|c|c|}
\hline Scientific Name & Common Name & Form & $\begin{array}{l}\text { Months } \\
\text { since } \\
\text { planting } \\
\text { (year } \\
\text { planted) }\end{array}$ & $\begin{array}{l}\text { Provenance (Kew science, } \\
\text { n.d.) }\end{array}$ \\
\hline Andropogon spp. & big bluestem & graminoid & n/a (2019) & $\mathrm{n} / \mathrm{a}$ \\
\hline Andropogon ternarius & splitbeard bluestem & graminoid & $84(2012)$ & $\begin{array}{l}\text { S., M.W., S.E. United } \\
\text { States, N.E. Mexico }\end{array}$ \\
\hline Andropogon virginicus & broomsedge bluestem & graminoid & $84(2012)$ & $\begin{array}{l}\text { E. North America (incl. } \\
\text { CA), N. South America }\end{array}$ \\
\hline Atriplex confertifolia & shadescale sagebrush & woody shrub & $113(2010)$ & $\begin{array}{l}\text { W. United States N.E. } \\
\text { Mexico }\end{array}$ \\
\hline $\begin{array}{l}\text { Bouteloua gracilis } \\
\text { 'Blonde Ambition' } \\
\text { (spontaneous) }\end{array}$ & blue grama grass & graminoid & $\mathrm{n} / \mathrm{a}(2019)$ & $\begin{array}{l}\text { C., M.W., S.W. United } \\
\text { States, Canada, Mexico* }\end{array}$ \\
\hline $\begin{array}{l}\text { Chrysanthemum } \\
\text { weyrichii }\end{array}$ & dwarf chrysanthemum & creeping forb & $101(2011)$ & C. Europe, C., N. Asia \\
\hline Ephedra minuta & miniature joint fir & woody shrub & $113(2010)$ & $\begin{array}{l}\text { S., C. China (China South- } \\
\text { Central, Qinghai) }\end{array}$ \\
\hline Erigeron vetensis & early bluetop fleabane & creeping forb & $113(2010)$ & M.W. United States \\
\hline Fallugia paradoxa & apache plume & woody shrub & $60(2014)$ & $\begin{array}{l}\text { S.W. United States, N. } \\
\text { Mexico }\end{array}$ \\
\hline $\begin{array}{l}\text { Genista tinctoria 'Royal } \\
\text { Gold' }\end{array}$ & $\begin{array}{l}\text { Dyer's greenweed, Dyer's } \\
\text { bloom }\end{array}$ & creeping forb & $60(2014)$ & Europe, Middle East \\
\hline Genista lydia & lydia broom & creeping forb & $36(2016)$ & S.E. Europe to Syria \\
\hline Ipomopsis aggregata & scarlet gilia & upright forb & $113(2010)$ & $\begin{array}{l}\text { S. W. United States (CO, } \\
\text { NM), N. Mexico }\end{array}$ \\
\hline Muhlenbergia montana & mountain muhly & graminoid & $84(2012)$ & $\begin{array}{l}\text { M.W. United States (incl. } \\
\text { CA), N., C. Mexico }\end{array}$ \\
\hline $\begin{array}{l}\text { Muhlenbergia spp. } \\
\text { (spontaneous) }\end{array}$ & muhly & graminoid & n/a (2019) & $\mathrm{n} / \mathrm{a}$ \\
\hline Silene schafta & autumn catchfly & creeping forb & $113(2010)$ & $\begin{array}{l}\text { Middle East (Iran, } \\
\text { Transcaucasia) }\end{array}$ \\
\hline Sporobolus heterolepis & prairie dropseed & graminoid & $\mathrm{n} / \mathrm{a}(2019)$ & M.W., N., E. United States \\
\hline $\begin{array}{l}\text { Teucrium graphaloides } \\
\text { (2) }\end{array}$ & germander & creeping forb & n/a (2019) & $\begin{array}{l}\text { S.W. Europe (Spain, } \\
\text { Portugal), Middle East }\end{array}$ \\
\hline
\end{tabular}

Note: * includes plants that are cultivars

Table 4. All taxa that 'perished' by scientific name, common name, plant growth form, months surviving (two dates means replanting), and provenance.

\begin{tabular}{|l|l|l|l|l|}
\hline & & $\begin{array}{l}\text { Months } \\
\text { alive since } \\
\text { Planting }\end{array}$ & \\
\hline
\end{tabular}




\begin{tabular}{|c|c|c|c|c|}
\hline Scientific Name & Common Name & Form & $\begin{array}{l}\text { (year } \\
\text { planted) }\end{array}$ & $\begin{array}{l}\text { Provenance (Kew } \\
\text { science, n.d.) }\end{array}$ \\
\hline Acantholimon acerosum & acantholimon, spike thrift & creeping forb, bun & $72(2008)$ & $\begin{array}{l}\text { Asia minor (Lebanon- } \\
\text { Syria, Turkey, } \\
\text { Transcaucasia), Middle } \\
\text { East }\end{array}$ \\
\hline Aethionema schistosum & fragrant Persian stonecress & creeping forb & $60(2007)$ & Asia minor (Turkey) \\
\hline Anacyclus maroccanus & Moroccan chamomile & creeping forb & $<12(2011)$ & N.W. Africa (Morocco) \\
\hline $\begin{array}{l}\text { Antennaria parvifolia } \\
\text { 'McClintock' }\end{array}$ & dwarf pussytoes & creeping forb & $60(2007)$ & $\begin{array}{l}\text { C. W. United States, } \\
\text { Canada, N. Mexico* }\end{array}$ \\
\hline Arenaria alfacarensis & Spanish sandwort & creeping forb & n/a (2007) & S.W. Europe (Spain) \\
\hline Artemisia sp. & wormwood & creeping forb & $\mathrm{n} / \mathrm{a}(2007)$ & Middle East \\
\hline Baileya multiradiata & desert marigold & upright forb & n/a (2009) & $\begin{array}{l}\text { S.W. United States, N. } \\
\text { Mexico }\end{array}$ \\
\hline Braya alpine & smooth rockcress & upright forb & $<12(2010)$ & $\begin{array}{l}\text { Central Europe (Austria, } \\
\text { Italy) }\end{array}$ \\
\hline Campanula incurva & Evia bellflower & upright forb & $24(2011)$ & $\begin{array}{l}\text { S. Europe (Greece, E. } \\
\text { Aegean Is.) }\end{array}$ \\
\hline Campanula kemulariae & bellflower, blue bells & upright forb & $<12(2010)$ & $\begin{array}{l}\text { Asia minor } \\
\text { (Transcaucasia) }\end{array}$ \\
\hline $\begin{array}{l}\text { Clematis columbiana } \\
\text { var. tenuiloba }\end{array}$ & rock clematis & creeping forb & $<12(2011)$ & $\begin{array}{l}\text { M.W., N.W. United } \\
\text { States }\end{array}$ \\
\hline Cotula hispida & silver cotula & creeping forb & $\begin{array}{l}72(2007) \\
60(2010)\end{array}$ & S. Africa \\
\hline Cotyledon orbiculata & pigs ear & succulent & $<12(2012)$ & S.W. Africa \\
\hline $\begin{array}{l}\text { Cytisus purgans } \\
\text { 'Spanish Gold' }\end{array}$ & Spanish gold broom & woody shrub & $96(2007)$ & $\begin{array}{l}\text { W. Europe (Spain, } \\
\text { France)* }\end{array}$ \\
\hline Delosperma cooperi & hardy ice plant & succulent & $96(2007)$ & $\begin{array}{l}\text { S. Africa (Free State, } \\
\text { Lesotho) }\end{array}$ \\
\hline Delosperma nubigenum & yellow ice plant & succulent & $96(2007)$ & $\begin{array}{l}\text { South Africa } \\
\text { (Free State, Lesotho) }\end{array}$ \\
\hline Delosperma 'Kelaidis' & Mesa Verde ice plant & succulent & $\begin{array}{l}72(2007) \\
84(2008)\end{array}$ & S. Africa* \\
\hline Draba streptocarpa & pretty draba & creeping forb, bun & n/a (2007) & $\begin{array}{l}\text { M.W. United States (CO, } \\
\text { NM, WY) }\end{array}$ \\
\hline $\begin{array}{l}\text { Dryas octopetala var. } \\
\text { hookeriana }\end{array}$ & Hooker's mountain-avens & creeping forb & $<12(2010)$ & $\begin{array}{l}\text { N.W., M.W. United } \\
\text { States, North America }\end{array}$ \\
\hline Erigeron elatior & tall fleabane & upright forb & $89(2011)$ & M.W. United States \\
\hline Erigeron leiomerus & rockslide yellow fleabane & creeping forb & $\mathrm{n} / \mathrm{a}(2011)$ & M.W. United States \\
\hline $\begin{array}{l}\text { Erysimum capitatum } \\
\text { var. purshii }\end{array}$ & alpine wallflower & upright forb & $24(2011)$ & $\begin{array}{l}\text { C. W. United States } \\
\text { N.W. North America }\end{array}$ \\
\hline $\begin{array}{l}\text { Euphorbia polychroma } \\
\text { 'Candy' }\end{array}$ & yellow cushion spurge & upright forb & $<12(2008)$ & $\begin{array}{l}\text { C., S.E. Europe, Asia } \\
\text { Minor* }\end{array}$ \\
\hline $\begin{array}{l}\text { Fendlera rupicola var. } \\
\text { wrightii }\end{array}$ & Wright's fendlerbush & woody shrub & $\mathrm{n} / \mathrm{a}(2007)$ & $\begin{array}{l}\text { S.W United States, N. } \\
\text { Mexico }\end{array}$ \\
\hline Festuca brachyphylla & alpine fescue & graminoid & $<12(2012)$ & $\begin{array}{l}\text { W., N. North America, } \\
\text { N. Asia, N. Europe } \\
\text { (Sweden) }\end{array}$ \\
\hline Hedeoma clone & pennyroyal & upright forb & $<12(2011)$ & S. United States (NM) \\
\hline Herniaria glabra & green carpet & creeping forb & n/a (2007) & $\begin{array}{l}\text { Europe, W. Asia, Middle } \\
\text { East, N. Africa, S. China }\end{array}$ \\
\hline Herniaria glabra'Sea & Sea foam green carpet & creeping forb & n/a (2008) & Europe, W. Asia, Middle \\
\hline
\end{tabular}




\begin{tabular}{|c|c|c|c|c|}
\hline Foam' & & & & $\begin{array}{l}\text { East, N. Africa, S. } \\
\text { China* }\end{array}$ \\
\hline Heteropappus sp. & Heteropappus & creeping forb & $<12(2010)$ & Eurasia \\
\hline Hypericum olympicum & $\begin{array}{l}\text { Mount Olympus St. John's } \\
\text { wort }\end{array}$ & upright forb & $48(2011)$ & $\begin{array}{l}\text { S. Europe, Asia Minor } \\
\text { (Turkey) }\end{array}$ \\
\hline Ipomopsis rubra & $\begin{array}{l}\text { standing cypress, scarlet } \\
\text { gilia }\end{array}$ & upright forb & $\mathrm{n} / \mathrm{a}(2008)$ & S. United States \\
\hline $\begin{array}{l}\text { Leucanthemum } \\
\text { atlanticum }\end{array}$ & $\begin{array}{l}\text { daisy (collected in } \\
\text { Morocco) }\end{array}$ & creeping forb & $<12(2011)$ & S., C. Europe \\
\hline Muhlenbergia emersleyi & bullgrass & graminoid & $36(2012)$ & $\begin{array}{l}\text { S.W. United States, } \\
\text { Mexico }\end{array}$ \\
\hline Olsynium biflorum & grass widow & upright forb & $<12(2011)$ & $\begin{array}{l}\text { S. South America (S. } \\
\text { Argentina) }\end{array}$ \\
\hline Panicum virgatum & switchgrass & graminoid & $96(2007)$ & $\begin{array}{l}\text { C., E. North America, } \\
\text { Mexico }\end{array}$ \\
\hline $\begin{array}{l}\text { Paronychia kapela } \\
\text { subsp. serpyllifolia }\end{array}$ & nailwort & creeping forb, bun & $\mathrm{n} / \mathrm{a}(2007)$ & S. Europe \\
\hline Penstemon angustifolius & broadbeard beardtongue & upright forb & $36(2010)$ & M.W. United States \\
\hline Penstemon caespitosus & mat penstemon & creeping forb & $72(2007)$ & $\begin{array}{l}\text { S. United States (WY, } \\
\text { CO, UT) }\end{array}$ \\
\hline Penstemon cyananthus & Wasatch beardtongue & upright forb & $24(2010)$ & $\begin{array}{l}\text { N.W. United States } \\
\text { (WY, MT, ID, UT) }\end{array}$ \\
\hline Penstemon fendleri & Fendler's penstemon & upright forb & $<12(2007)$ & $\begin{array}{l}\text { S. United States, N.E. } \\
\text { Mexico }\end{array}$ \\
\hline Penstemon grahamii & Graham beardtongue & creeping forb & $<12(2010)$ & $\begin{array}{l}\text { W. United States (CO, } \\
\text { UT) }\end{array}$ \\
\hline $\begin{array}{l}\text { Penstemon linarioides } \\
\text { subsp. coloradensis }\end{array}$ & $\begin{array}{l}\text { Colorado narrowleaf } \\
\text { beardtongue }\end{array}$ & creeping forb & $60(2007)$ & S.W. United States \\
\hline $\begin{array}{l}\text { Petrophytum } \\
\text { caespitosum }\end{array}$ & mat rockspirea & creeping forb & $<12(2010)$ & $\begin{array}{l}\text { W. United States. N.E. } \\
\text { Mexico }\end{array}$ \\
\hline $\begin{array}{l}\text { Phacelia campanularia } \\
\text { (seed) }\end{array}$ & California bluebells & upright forb & $<12(2009)$ & W. United States (CA) \\
\hline Poa fendleriana & muttongrass & graminoid & $36(2012)$ & $\begin{array}{l}\text { W. United States, N. } \\
\text { Mexico, W. Canada }\end{array}$ \\
\hline Potentilla hyparctica & arctic cinquefoil & creeping forb & $\mathrm{n} / \mathrm{a}(2007)$ & $\begin{array}{l}\text { N. Asia, N. Europe } \\
\text { (Sweden), N.W., N.E. } \\
\text { North America }\end{array}$ \\
\hline $\begin{array}{l}\text { Potentilla nepalensis } \\
\text { 'Shogran' }\end{array}$ & Nepal cinquefoil & creeping forb & $<12(2010)$ & E. Asia, W. Himalayas* \\
\hline Putoria calabrica & stinking madder & upright forb & $<12(2011)$ & $\begin{array}{l}\text { S. Europe, Asia minor, } \\
\text { N. Africa }\end{array}$ \\
\hline Salvia cryptantha & salvia & upright forb & $<12(2011)$ & Asia minor (Turkey) \\
\hline Salvia 'Eskihar' & Eskihar sage & creeping forb & $<12(2011)$ & Asia minor* \\
\hline Scutellaria prostrata & prostrate skullcap & creeping forb & $<12(2011)$ & Himalayas \\
\hline Scutellaria scordifolia & skullcap hyssop & creeping forb & $<12(2010)$ & S. Mexico \\
\hline Sedum rupestre & blue stonecrop & succulent & $48(2011)$ & Europe, Asia minor \\
\hline Streptanthus cordatus & heartleaf twistflower & creeping forb & $36(2010)$ & W. United States \\
\hline Teucrium aroanium & gray creeping germander & creeping forb & $53(2010)$ & S. Europe (Greece) \\
\hline Teucrium graphaloides & germander & creeping forb & $65(2011)$ & $\begin{array}{l}\text { S.W. Europe (Spain, } \\
\text { Portugal), Middle East }\end{array}$ \\
\hline Thymus neiceffi & juniper leaf thyme & creeping forb & $108(2007)$ & Europe, N. Africa, Asia \\
\hline
\end{tabular}




\begin{tabular}{|l|l|l|l|l|}
\hline & & & & \\
\hline Townsendia eximia & Townsend's daisy & upright forb & n/a (2007) & $\begin{array}{l}\text { S.W. United States (CO, } \\
\text { N.M) }\end{array}$ \\
\hline Vella spinosa & fine broom, spiny broom & creeping forb & $<12(2011)$ & S. Europe (Spain) \\
\hline Ziziphora bungeana & Ziziphora & upright forb & $<12(2011)$ & Middle East, C. Asia \\
\hline
\end{tabular}

Note: * includes plants that are cultivars

\section{Data Collection and Analysis}

Each year in early autumn, data were collected on each of the plant taxa on the green roof. Plants were observed and recorded in the categories of survive or perish, and when possible, the number of remaining plants were recorded. Additionally, in April 2020 data was collected on the number of individual plants that have overwintered for each taxa. This was then used to determine the calendar months of survival and the relative percent survival rate for each taxa since planting. Additionally, data on plant provenance was noted to help interpret success rate of these taxa on a green roof in a semi-arid climate.

\section{RESULTS AND DISCUSSION}

\section{Succulents}

The succulent category had the overall highest survival rate in this evaluation (Figure 2). This is not surprising as succulents have proven to be successful on green roofs around the globe (Erwin et al., 2019; Nektarios et al., 2015; Vahdati et al., 2017) with some exceptions (Nektarios et al., 2020). From 2007-2009, 19 succulent taxa were planted (Hesperaloe parviflora and Delosperma 'Kelaidis' planted twice). Sixteen out of 19 taxa, or $84 \%$, have survived. Later, between 2010-2012, three succulent taxa were planted, and of them, one, or $33 \%$, survived. In total, 22 succulent taxa have been trialed on the rooftop, of them, 17, or $77 \%$, have survived.

Many of the succulents on the DBG green roof have been successful and grown nearly exponentially, including: Agave parryi, Nolina microcarpa, Opuntia aurea, Opuntia phaeacantha, Opuntia polyacantha, Phedimus kamtschaticus Phemeranthus calycinus, and $S$. lanceolatum. Four of the total six succulents that perished did so in 2015, all likely due to a sudden November 2014 freeze, including S. rupestre, D. cooperi, D. nubigenum, and one of the two $D$. 'Kelaidis'. This suggests that the genus Delosperma may not be suitable for our lowirrigation, semi-arid, temperate green roof; all the species trialed in the genus perished in this evaluation. Remarkably, others have found the genus Delosperma to be resilient and ideal for use in water limited green roofs (Bousselot et al,. 2010; Bousselot et al., 2011; Klein and Coffman 2015; Thuring et al., 2010).

Although less evaluated on green roofs in general, the monocarpic A. parryi has shown incredible vigor. Starting from four plants in 2008, with two blooming in 2011, it has resulted in 19 plants between both reseeding and runners, increasing in individuals by $475 \%$. Maihuenia poeppigii has survived, although it was observed as perished in 2013 and 2014; this likely due to early fall dormancy or its branched, dry aesthetic. Cotyledon orbiculata, which was mistakenly installed by a helper on the green roof, quickly perished the year it was planted, 
2012, as soon as it became cold. C. orbiculata is only hardy to zone 9b-12, making it too tender for survival outdoors in Colorado in USDA hardiness zone 5.

Interestingly, of all 22 succulent taxa that were planted, all 15 that are native to North America have survived. Only two have survived that are not native to the United States: M. poeppigii, native to South America and P. kamtschaticus, native to Asia. Of the five succulent taxa that have perished, four are native to Africa: C. orbiculata, D. cooperi, D. 'Kelaidis', and $D$. nubigenum. This may indicate that Delosperma species native to Africa are not as likely to survive on a USDA plant hardiness zone 5 low-water green roof in a semi-arid, temperate climate in the United States. The other succulent to perish, S. rupestre, is native to Europe.

\begin{tabular}{|c|c|c|c|c|c|c|c|c|c|c|c|c|c|c|c|}
\hline \multicolumn{16}{|c|}{ Succulent Plant Taxa Survival Chart } \\
\hline Plant Taxa & 2007 & 2008 & 2009 & 2010 & 2011 & 2012 & 2013 & 2014 & 2015 & 2016 & \begin{tabular}{|l|l|}
52017 \\
\end{tabular} & \begin{tabular}{l|l|}
72018 \\
\end{tabular} & 2019 & 2020 & Survival \% \\
\hline Agave paryi & & 4 & & & 3 & 3 & 2,6 & 2,6 & & & & & 19 & 19 & $475 \%$ \\
\hline Echinocereus fendleri & & & mass & & 6 & 6 & & & & & & & & 4 & $n / a$ \\
\hline Echinocereus triglochidiatus & 18 & & & & 18 & 18 & 18 & 18 & & & & & & 4 & $22 \%$ \\
\hline $\begin{array}{l}\text { Echinocereus triglochidiatus } \\
\text { white sand strain }\end{array}$ & 2 & & & & 2 & 2 & 2 & 2 & & & & & & 1 & $50 \%$ \\
\hline Echinocereus viridiflorus & 11 & & & & 8 & 8 & & & & & & & & 2 & $18 \%$ \\
\hline Escobaria missouriensis & mass & & & & 2 & 6 & 3 & 3 & & & & & & 3 & $\mathrm{n} / \mathrm{a}$ \\
\hline Escobaria vivipara & 20 & & & & 20 & 9 & 3 & 3 & & & & & & 3 & $15 \%$ \\
\hline Hesperaloe parviflora & 8 & & & & 8 & 8 & 6 & 6 & & & & & & 4 & $50 \%$ \\
\hline Hesperaloe parviflora (2) & & 5 & & & 5 & 5 & 3 & 3 & & & & & & 4 & $80 \%$ \\
\hline Maihuenia poeppigii & & 27 & & & $\frac{6}{6}$ & 6 & 0 & 0 & & $\sim$ & $\sim$ & $\sim$ & & $\sim$ & $n / a$ \\
\hline Nolina microcarpa & 3 & & & & 6 & 6 & 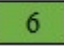 & 6 & & & & & & 5 & $167 \%$ \\
\hline Opuntia aurea & mass & & & & mass & mass & mass & mass & & & & & & mass & $n / a$ \\
\hline Opuntia phaeacantha & mass & & & & mass & mass & mass & mass & & & & & & mass & $n / a$ \\
\hline Opuntia polyacantha & mass & & & & mass & mass & mass & mass & & & Key & & & mass & n/a \\
\hline Pediocactus simpsonii & 10 & & & & 10 & 5 & 3 & 3 & & & $\frac{\text { surv }}{\text { Seec }}$ & & & & $\mathrm{n} / \mathrm{a}$ \\
\hline Phedimus kamtschaticus & 5 & & & & mass & mass & mass & mass & & & Peris & & & mass & $\mathrm{n} / \mathrm{a}$ \\
\hline Phemeranthus calycimus & & & & & & & & & & & Unkr & nown & & mass & $n / a$ \\
\hline Phemeranthus calycimus (2) & & & & & 12 & & & & & & & & & mass & $n / a$ \\
\hline Sedum lanceolatum & 64 & & & & mass & mass & mass & mass & & & & & & mass & $\mathrm{n} / \mathrm{a}$ \\
\hline Cotyledon orbiculata & & & & & & 5 & & & & & & & & & $0 \%$ \\
\hline Delosperma cooperi & 12 & & & & mass & mass & 6 & 6 & 0 & & & & & & $0 \%$ \\
\hline Delosperma 'Kelaidis' & 40 & & & & 2 & 2 & 0 & & & & & & & & $0 \%$ \\
\hline Delosperma 'Kelaidis' (2) & & 8 & & & 2 & 2 & 2 & 2 & 0 & & & & & & $0 \%$ \\
\hline Delosperma nubigenum & 21 & & & & mass & mass & 2 & 2 & 0 & & & & & & $0 \%$ \\
\hline Sedum rupestre & & & & & 31 & mass & mass & mass & 0 & & & & & & $0 \%$ \\
\hline
\end{tabular}

Figure 2. Succulent survival by year from 2007-2020. The plants categorized as 'survive' are listed alphabetically by genus at the top while those that are categorized as 'perish' are listed alphabetically by genus at the bottom. Note: \#,\# second number represents number of pups, 'mass' = many individual plants that have grown together over time. 


\section{Creeping Forbs}

In the first two years after the green roof was installed, fall 2007-September 2009, 26 creeping forb taxa were planted (with Eriogonum umbellatum var. aureum being planted twice but counted as one taxa; Figure 3). After 12 years, 12 of the 26 plant taxa have survived, or $46 \%$. Over the next two planting seasons, 2010-2012, 19 creeping forbs were planted (including a second Cotula hispida, therefore, only 18 'new' taxa were planted). Of the 19 creeping forb taxa only 3, or 16\% survived. Between the seasons of 2013-2018 two taxa were planted, Genista tinctoria and G. lydia, both of which have survived. In the past 12 years (2007-September 2019) 17 of the total 47 species, or $36 \%$, have survived. C. hispida, E. umbellatum var. aureum, and G. tinctoria were trialed twice but were only counted as taxa once. Both plantings of $C$. hispida have perished, both E. umbellatum var. aureum survived, and G. tinctoria (2) was planted in 2019 therefore only data on the first planted G. tinctoria is included in the data as perished.

Since the original evaluation in 2012, seven years later, seven more creeping forb plant taxa have perished; Acantholimon acerosum, Aethionema schistosum, Artemisia sp., C. hipida, Penstemon caespitosus, Streptanthus cordatus and Thymus neiceffi (Schneider et al., 2014). Most form taproots, which have previously been shown to be less ideal for extensive green roofs (Nagase and Dunnett 2010). Many creeping forbs trialed on the green roof can reseed which has made tracking them more difficult, although that could be a factor in overall plant species success. In the original evaluation, Erigeron vetensis, Heterotheca jonesii, and Veronica pectinata were marked as having perished, but since, has reappeared through seeding. $E$. vetensis has declined in number but has had great overall success and has bloomed again (authors observation, April 2020). E. leiomerus was planted in 2011, perished between $2012-$ 2014 , then appeared in 2015, surviving for five years until perishing again. T. neiceffi survived on the green roof for nine years before perishing and should be reevaluated.

Of the subcategory of bun forming creeping forbs, three of the six, or $50 \%$, have survived. $A$. armenum, A. litvinovii, and Dianthus anatolicus have been successful in survival, surviving for 11 total years so far. Although technically successful, each species has declined in number without showing any remarkable new growth. This confirms what was stated in the original study, the small size and slow growth of bun forming creeping forbs may be unfavorable for covering large areas. A. acerosum survived for six years before perishing and could be retried as its two relatives $A$. armenum and $A$. litvinovii have been successful.

Of the 47 trialed creeping forb plant taxa, 16 are native to the United States. Of the 16 that are native, seven have survived, or $44 \%$. Thirty-one of the trailed plants are non-native, of them, 13 survived, or $42 \%$. Despite the results being almost equal, creeping forbs that are native to this country, and especially our ecoregion, theoretically should be more likely to survive these harsh conditions, however more evaluation would be required. 


\begin{tabular}{|c|c|c|c|c|c|c|c|c|c|c|c|c|c|c|c|}
\hline \multicolumn{16}{|c|}{ Creeping Forbs Plant Taxa Survival Chart } \\
\hline Plant Taxa & 2007 & 2008 & 2009 & 2010 & 2011 & 2012 & 2013 & 2014 & 2015 & 2016 & 2017 & 2018 & 2019 & 2020 & Survival \% \\
\hline Acantholimon armenum & & 5 & & & 1 & 1 & 1 & 1 & & & & & 1 & 1 & $20 \%$ \\
\hline Acantholimon litvinovii & & 5 & & & 1 & 1 & 1 & 1 & 2 & & & & & 1 & $20 \%$ \\
\hline Chrysanthemum weyrichii & & & & & 16 & mass & mass & mass & & $\sim$ & $\sim$ & & & 1 & $6 \%$ \\
\hline Dianthus anatolicus & & 28 & 19 & & 10 & 11 & 17 & 17 & 10 & & & & 10 & 10 & $36 \%$ \\
\hline Draba hispanica & 3 & & & & 10 & mass & & & & & & & $\sim$ & 1 & $33 \%$ \\
\hline Erigeron vetensis & & & & 27 & & 0 & & & & & & & & 6 & $22 \%$ \\
\hline $\begin{array}{l}\text { Eriogomum umbellatum var. } \\
\text { aureum }\end{array}$ & 15 & & 12 & & 9 & 9 & 9 & 9 & 2 & & & & & 3 & $20 \%$ \\
\hline $\begin{array}{l}\text { Eriogomum umbellatum var. } \\
\text { aureum (2) }\end{array}$ & & 10 & & & 6 & 9 & mas & mass & 2 & & & & 2 & 2 & $20 \%$ \\
\hline Genista tinctoria 'Royal Gold' & & & & & & & & 1 & & & & & 1 & 3 & $300 \%$ \\
\hline Genista lydia & & & & & & & & & & 1 & & & & 4 & $400 \%$ \\
\hline Heterotheca jonesii & 32 & & & & & 0 & & & & & & & & mass & $\mathrm{n} / \mathrm{a}$ \\
\hline Penstemon pinifolius & & & 6 & & 18 & 5 & 5 & 5 & & & & & & mass & $\mathrm{n} / \mathrm{a}$ \\
\hline Phlox bifida 'Betty Blake' & 1 & & & & 1 & 1 & 5 & 5 & & & & & & 1 & $100 \%$ \\
\hline Physaria bellii & 64 & & & & 17 & & & & & & & & & 7 & $11 \%$ \\
\hline Silene schafta & & & & 23 & 10 & 10 & $10+$ & $10+$ & & & & & & 7 & $30 \%$ \\
\hline Tencrium graphaloides (2) & & & & & & & & & & & & & 3 & 3 & $100 \%$ \\
\hline Veronica liwanensis & 9 & & & & 5 & 5 & 7 & 8 & & & & & & 2 & $22 \%$ \\
\hline Veronica pectinata & mass & & & & 0 & L & 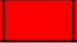 & 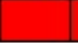 & & & & & & 2 & $\mathrm{n} / \mathrm{a}$ \\
\hline Veronica thymodies & 13 & & & & 6 & 6 & 6 & 6 & & & & & & 1 & $8 \%$ \\
\hline Acantholimon acerosum & & 4 & & & 3 & 2 & 2 & 1 & 0 & & & & & & $0 \%$ \\
\hline Aethionema schistosum & 3 & & & & 3 & 1 & 0 & & & & & & & & $0 \%$ \\
\hline Anacychus maroccamus & & & & & 6 & 0 & & & & & & & & & $0 \%$ \\
\hline $\begin{array}{l}\text { Antennaria parvifolia } \\
\text { 'McClintock' }\end{array}$ & 64 & & & & 1 & 0 & & & & & & & & & $0 \%$ \\
\hline Arenaria alfacarensis & 16 & & & & 0 & & & & & & & & & & $0 \%$ \\
\hline Artemisia sp. & 32 & & & & & 10 & & & & & & & & & $0 \%$ \\
\hline $\begin{array}{l}\text { Clematis collumbiana var. } \\
\text { temuiloba }\end{array}$ & & & & & 2 & 0 & & & & & & & & & $0 \%$ \\
\hline Cotula hispida & 9 & & & & mass & mass & 0 & & & & & & & & $0 \%$ \\
\hline Cotula hispida (2) & & & & 21 & 10 & 20 & mass & mass & 0 & & & & & & $0 \%$ \\
\hline Draba streptocarpa & 32 & & & & 0 & & & & & & & & & & $0 \%$ \\
\hline $\begin{array}{l}\text { Diyas octopetala var. } \\
\text { hookeriana }\end{array}$ & & & & 10 & 0 & & & & & & & & & & $0 \%$ \\
\hline
\end{tabular}




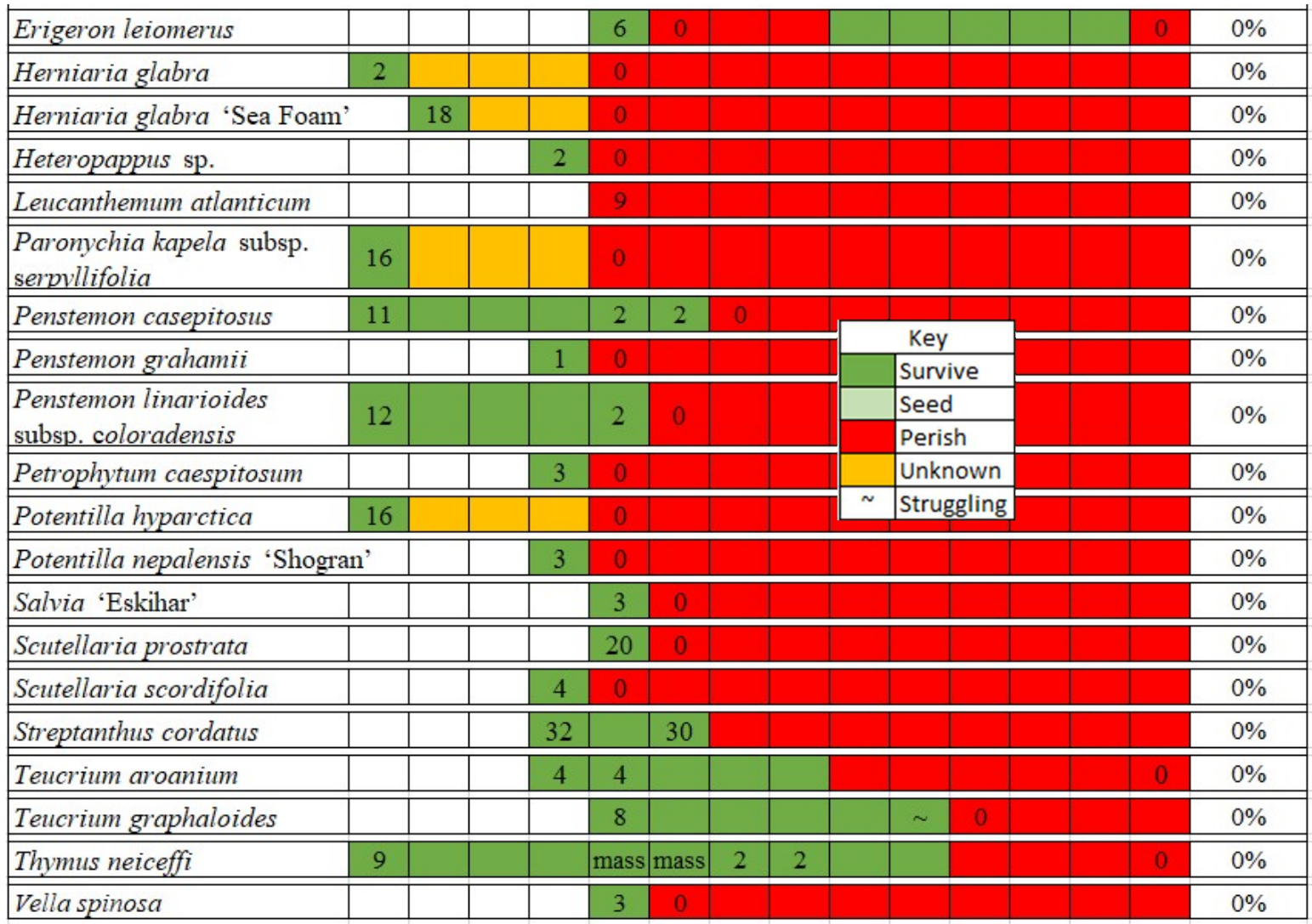

Figure 3. Creeping forbs survival by year from 2007-2020. The plants categorized as 'survive' are listed alphabetically by genus at the top while those that are categorized as 'perish' are listed alphabetically by genus at the bottom.

\section{Upright Forbs}

To date, a total of 24 upright forb taxa have been trialed on the DBG green roof (Figure 4). From installation in falls 2007-2009, ten total upright forb taxa were planted. Of the ten, four, or $40 \%$ survived. Between fall 2010-2012, 14 more upright forbs were planted for trial and out of the 14 planted only one, or $7 \%$, survived. The total survival rate for upright forb taxa on the green roof is five of 23 , or $22 \%$.

As previously found in the five-year evaluation (Schneider et al., 2014), most upright forbs in low-irrigation green roofs have limited use in semi-arid climates. However, five of the plant taxa, Echium amoenum, Ipomopsis aggregata, Iris germanica dwarf, Salvia pachyphylla, and Thelesperma ambiguum have survived 12 years, demonstrating that upright forb taxa should be considered for semi-arid environments on green roofs after careful trial. Note: Hypericum olympicum in the 2014 evaluation was listed as woody shrub (Schneider et al., 2014). In this evaluation it is considered an upright forb as it performs more as a perennial than a subshrub in Colorado as it does not have above ground woody material that overwinters.

In this evaluation, the upright forb category has had the lowest success rate for semi-arid climates on a green roof. Of the 17 perished upright forbs, 11 taxa perished within one year then all the rest except E. elatior and H. olympicum have perished within three years of planting. 
This suggests that these upright forbs that are not adapted to semi-arid, temperate green roofs will perish quickly. E. amoenum, I. aggregata, and I. germanica dwarf are quite successful and have grown exponentially, eventually massing. From the results of this evaluation, these species are recommended for use on semi-arid green roofs.

Erigeron elatior and S. pachyphylla have consistently survived, however, E. elatior has perished in 2020, although both were mistakenly marked as perished in the original study. This may be due to their appearance, such as early dormancy, when they were counted in the fall (Schneider et al. 2014). E. elatior survived for eight years before eventually perishing; it is an alpine, therefore, premature die back is normal. However, the species that perished may have done so due to the lack of winter snow cover and nighttime temperatures that reached $-1^{\circ} \mathrm{C}$ to $4^{\circ} \mathrm{C}$.

Although three $S$. pachyphylla were originally planted during the installation of the green roof in 2007, only one has remained. Baileya multiradiata seeds were sown in 2009 but did not germinate until 2016 when it appeared for two years then perished; this lifecycle is normal as $B$. multiradiata is biennial, but it is unknown if the plant flowered before dying. In general, $B$. multiradiata germination is erratic which could play a role in their low germination rate in this evaluation.

On the DBG green roof, T. ambiguum has survived for 12 years but is now showing a decline in number, although it has varied in its relative vigor seasonally, showing potential for its use for green roofs in semi-arid climates. As discussed in the original study, Ipomopsis species reseed which allow them to move around the roof to areas that provide ideal conditions (Schneider et al. 2014); Overall, their reseeding could allow them to be more successful than non-seeding upright forbs. Originally I. rubra was planted on the green roof in 2007 and did survive and reseed up until the first evaluation (Schneider et al, 2014) but then eventually died out to be replaced entirely by its close relative, I. aggregata. While I. aggregata was first planted in 2010 , this biennial quickly flowered, reseeded and continued to dominate the shallow portions of the green roof. One of the surviving plants, I. germanica dwarf develops underground rhizomes which may benefit its survival due to its horizonal growth habit.

Provenance in the upright forbs category include taxa ranging from North America to southeastern Asia. Of the surviving seven upright forbs, five, or $71 \%$, are native to the United States. The other two surviving taxa are E. amoenum, which is native to the Middle East, and $I$. germanica dwarf, native to southeastern Europe. Of the taxa that perished, eight of the 16, or $50 \%$, are native to the United States. The data from this evaluation suggests that upright forbs that are native to our continent may be more likely to survive on a semi-arid green roof, although further evaluation is necessary. 


\begin{tabular}{|c|c|c|c|c|c|c|c|c|c|c|c|c|c|c|c|}
\hline \multicolumn{16}{|c|}{ Upright Forbs Plant Taxa Survival Chart } \\
\hline Plant Taxa & 2007 & 2008 & 2009 & 2010 & 2011 & 2012 & 2013 & 2014 & 2015 & 2016 & 62017 & 2018 & 2019 & 2020 & Survival \% \\
\hline Echium amoenum & 4 & & & & 4 & 4 & 4 & 4 & & & & & 8 & mass & $n / a$ \\
\hline Iris germanica dwarf & 26 & & & & 26 & 26 & & & & & & & & mass & $\mathrm{n} / \mathrm{a}$ \\
\hline Ipomopsis aggregata & & & & 18 & & & & & & & & & & mass & $\mathrm{n} / \mathrm{a}$ \\
\hline Salvia pachyphylla & 3 & & & & 1 & 1 & 1 & 1 & & & & & 1 & 1 & $n / a$ \\
\hline Thelesperma ambiguum & 32 & & & & mass & mass & & & & & & & & 3 & $n / a$ \\
\hline Baileya multiradiata & & & seed & 0 & & & & & & $\sim$ & $\sim$ & 0 & & & $0 \%$ \\
\hline Braya alpine & & & & 12 & 0 & & & & & & & & & & $0 \%$ \\
\hline Campanula incurva & & & & & 6 & 2 & 0 & & & & & & & & $0 \%$ \\
\hline Campanula kemulariae & & & & 1 & 0 & & & & & & & & & & $0 \%$ \\
\hline Erigeron elatior & & & & & 8 & 8 & 8 & 8 & & & & & & 0 & $0 \%$ \\
\hline $\begin{array}{l}\text { Erysimum capitatum var. } \\
\text { purshii }\end{array}$ & & & & & 31 & 28 & 0 & & & & & & & & $0 \%$ \\
\hline $\begin{array}{l}\begin{array}{l}\text { Euphorbia polychroma } \\
\text { 'Candy' }\end{array} \\
\end{array}$ & & mass & & & & & & & & & & & & & $0 \%$ \\
\hline Hedeoma clone & & & & & 3 & 0 & & & & & & & & & $0 \%$ \\
\hline Hypericum olympicum & & & & & 4 & 4 & $4+$ & $4+$ & 0 & & & & & & $0 \%$ \\
\hline Ipomopsis rubra & & 16 & & & & & & & & 0 & & & & & $0 \%$ \\
\hline Olsynium biflorum & & & & & 4 & 0 & & & & & & & & & $0 \%$ \\
\hline Penstemon angustifolius & & & & 2 & 1 & 1 & 0 & & & & & & & & $0 \%$ \\
\hline Penstemon cyananthus & & & & 6 & 6 & 0 & & & & & & & & & $0 \%$ \\
\hline Penstemon fendleri & 8 & 0 & & & & & & & & & Key & & & & $0 \%$ \\
\hline Phacelia campanularia & & & seed & 0 & & & & & & & Seed & & & & $0 \%$ \\
\hline Putoria calabrica & & & & & 32 & 0 & & & & & Perish & & & & $0 \%$ \\
\hline Salvia cryptantha & & & & & 5 & 0 & & & & $\sim$ & & & & & $0 \%$ \\
\hline Townsendia eximia & 24 & & & & 0 & & & & & & . & & & & $0 \%$ \\
\hline Ziziphora bungeana & & & & & 17 & 0 & & & & & & & & & $0 \%$ \\
\hline
\end{tabular}

Figure 4. Upright forbs survival by year from 2007-2020. The plants categorized as 'survive' are listed alphabetically by genus at the top while those that are categorized as 'perish' are listed alphabetically by genus at the bottom.

\section{Graminoids}

A total of 11 graminoid species have been trialed on the DBG green roof (Figure 5). Out of the 11 , four of them have been planted or seeded in 2019, therefore will not be included in the overall survival rate. Between fall 2007- 2009 one graminoid was planted, Panicum virgatum, which survived eight years before perishing. Between fall 2010-2012, six more graminoids were trialed. Of the six, three survived, or 50\%. The surviving graminoids planted between 2007 2019 include $A$. ternaries, A. virginicus, and M. montana, each of them have survived for seven years, showing great potential. Graminoids overall (excluding plantings in 2019) have a three of seven, or $43 \%$ survival rate. This indicates that specific graminoid plant taxa should continue to be trialed to determine successful candidates for semi-arid green roofs. As of April 2020, all graminoids planted in 2019 have survived but will need a longer evaluation period to determine their overall success. 
Of the graminoids on the green roof, $A$. ternarius had the highest survival at $40 \%$, followed by $A$. virginicus as a close second. All three of the surviving graminoids have decreased in number, M. montana more than the others, starting with 64 plants and as of 2019 only three are surviving. Although all have decreased in number, the graminoids that remain are showing great potential.

Our data suggest that the genus Festuca is not a good candidate for low water green roofs in high elevation, semi-arid climates. Sixty-four $F$. brachyphylla were planted and all perished within a year. Similarly, an evaluation in arid Iran found two relatives, $F$. aurundinacea and $F$. ovina, to be unsuccessful (Vahdati et al., 2017) although Nektarios et al. (2014) reported that $F$. aurndinaceae was a successful green roof plant with irrigation. Erwin et al. (2019) also found that three Festuca spp. performed well.

$P$. virgatum survived for eight years on the DBG roof top and suddenly perished in 2015 yet thrived for Erwin et al. (2019). This could be due to differences in climate or substrate depth, although $P$. virgatum should be re-trialed to confirm this conclusion. It is speculated that $P$. virgatum, along with M. emersleyi and Poa fendleriana, suddenly perished in 2015 because of a November freeze in 2014 (author observation). And it is suspected that they would have otherwise survived.

Sporobolus heterolepis, planted in 2019, has been established for one year and has been successful in producing another plant. The success of $S$. heterolepis on the DBG green roof contrasts with what was found by Tran et al. (2019) that this graminoid did not thrive with lower precipitation levels. Bouteloua gracilis made a spontaneous appearance in 2019 and was successful overwintering.

\begin{tabular}{|c|c|c|c|c|c|c|c|c|c|c|c|c|c|c|c|}
\hline \multicolumn{16}{|c|}{ Graminoids Plant Taxa Survival Chart } \\
\hline Plant Taxa & 2007 & 2008 & 2009 & 2010 & 2011 & 2012 & 2013 & 2014 & 2015 & 2016 & 2017 & 2018 & 2019 & 2020 & Survival \% \\
\hline Andropogon ternarius & & & & & & 10 & 4 & 4 & & & & & 3 & 4 & $40 \%$ \\
\hline Andropogon virginicus & & & & & & 10 & 7 & 7 & & & & & 3 & 3 & $30 \%$ \\
\hline Andropogon sp. & & & & & & & & & & & & & 3 & & $n / a$ \\
\hline Bouteloua gracilis 'Blonde & & & & & & & & & & & & & & & \\
\hline Ambition' & & & ey & & & & & & & & & & 1 & 1 & $100 \%$ \\
\hline Muhlenbergia montana & & & Irvive & & & 64 & 64 & 64 & 5 & & & 3 & 3 & 3 & $5 \%$ \\
\hline Muhlenbergia sp. & & & erish & & & & & & & & & & 1 & & $\mathrm{n} / \mathrm{a}$ \\
\hline Sporobolus heterolepis & & & nnow & & & & & & & & & & 2 & 3 & $150 \%$ \\
\hline Festuca brachyphylla & & & rugglir & & & 64 & 0 & & & & & & & & $0 \%$ \\
\hline Muhlenbergia emersleyi & & & & & & 10 & 10 & 10 & 0 & & & & & & $0 \%$ \\
\hline Panicum virgatum & 6 & & & & 6 & 6 & 6 & 6 & 0 & & & & & & $0 \%$ \\
\hline Poa fendleriana & & & & & & 64 & 2 & 2 & 0 & & & & & & $0 \%$ \\
\hline
\end{tabular}

Figure 5. Graminoids survival by year from 2007-2020. The plants categorized as 'survive' are listed alphabetically by genus at the top while those that are categorized as 'perish' are listed alphabetically by genus at the bottom. 


\section{Woody Shrubs}

In this evaluation, the woody shrubs were planted on or near the berm area on the green roof where substrate reaches its maximum depth of around $45 \mathrm{~cm}$. Although less represented in literature, these taxa have shown good overall survivability in our study (Figure 6). Between 2007-2009, 12 taxa were planted, of the 12 planted, ten, or $83 \%$, survived. Two taxa were planted between 2010-2012 and one of the two, or 50\%, survived. In 2014, Fallugia paradoxa appeared and has continued to survive. Of the total 15 woody shrub taxa, 13, or $87 \%$, have survived. Overall, woody shrubs show great potential for use on green roofs in a semi-arid climate.

One of the most remarkable observations on this green roof was the spontaneous arrival of $F$. paradoxa. It appeared in 2014 and has become established, blooming, and producing seed. S. greggii was the most successful woody shrub (technically a subshrub) on this semi-arid green roof, growing into a mass from 5 original plants throughout 11 years. The green roof has proven to be an ideal environment for this plant taxa sharing similar qualities to its natural environment such as high light and low moisture. Chamaebatiaria millefolium has also been quite successful, reseeding and doubling the original number of plants. This is likely due to its adapted xeric properties.

Although Ericameria nauseosa subsp. nauseosa var. nauseosa's survival rate is listed as $80 \%$, it has been vigorous and has seeded several times (some specific years unknown). It has emerged throughout the rooftop and is continuously being removed by the gardener seasonally. Cercocarpus breviflorus and Chilopsis linearis reappear seasonally and bloom. The surviving plants have maintained a steady population annually. No taxa have declined more than $50 \%$ and seem to maintain a steady number annually. One exception is $A$. confertifolia, at $17 \%$. Despite their high success rate, woody shrubs are seldom mentioned in literature; this study suggests that they are candidates for further evaluation.

The data suggest that provenance may play a role on these taxa's survivability in a semi-arid climate. Eleven of the 13 surviving taxa, or $85 \%$, are native to the United States. The other two surviving taxa, Ephedra minuta and Euonymus nanus var. turkestanicus are native to Asia. More shrubs native to Asia should be trialed to determine if provenance is a determining factor on success rate. Of the two taxa that perished, Cytisus purgans 'Spanish Gold' is native to Europe, and the other one, Fendlera rupicola var. wrightii, is native to the United States. The data collected suggests that provenance could be a factor in the success rate of woody plant taxa on green roofs. 


\begin{tabular}{|c|c|c|c|c|c|c|c|c|c|c|c|c|c|c|c|}
\hline \multicolumn{16}{|c|}{ Woody Shrubs Plant Taxa Survival Chart } \\
\hline Plant Taxa & 2007 & 2008 & 2009 & 2010 & \begin{tabular}{|l|}
2011 \\
\end{tabular} & 2012 & 2013 & 2014 & 2015 & 2016 & $6|2017|^{2}$ & 2018 & 2019 & 2020 & Survival \% \\
\hline Amorpha fruticosa 'Nana' & & & 3 & & 3 & 4 & 3 & 3 & 3 & & & & 3 & 3 & $100 \%$ \\
\hline Atriplex confertifolia & & & & 6 & 6 & 2 & & & 1 & 1 & 1 & & & 1 & $17 \%$ \\
\hline $\begin{array}{l}\text { Arctostaphylos 'Lauren's } \\
\text { Best' }\end{array}$ & 1 & & & & 1 & 1 & 1 & 1 & & & & & & 1 & $100 \%$ \\
\hline Arctostaphylos patula & 3 & & & & 2 & 2 & 2 & 2 & & & & & & 2 & $67 \%$ \\
\hline $\begin{array}{l}\text { Arctostaphylos } \mathrm{x} \\
\text { coloradoensis 'Cascade' }\end{array}$ & 2 & & & & 1 & 2 & 2 & 2 & & & & & & 1 & $50 \%$ \\
\hline Cercocarpus breviflorus & 3 & & & & 3 & 3 & 3 & 3 & & & & & & 3 & $100 \%$ \\
\hline Chamaebatiaria millefolium & 3 & & & & 3 & 3 & 3 & 3 & & & & & 7 & 6 & $200 \%$ \\
\hline Chilopsis linearis & 2 & & & & 2 & 2 & 2 & 2 & 1 & & & & 1 & 1 & $50 \%$ \\
\hline Ephedra minuta & & & & 6 & 6 & 5 & 6 & 6 & & & & & & 3 & $50 \%$ \\
\hline $\begin{array}{l}\text { Ericameria nauseosa subsp. } \\
\text { nauseosa var. nauseosa }\end{array}$ & 5 & & & & & & 1 & 1 & & & $\begin{array}{l}\text { Key } \\
\text { urvive }\end{array}$ & & & 4 & $80 \%$ \\
\hline $\begin{array}{l}\text { Euonymus nanus var. } \\
\text { turkestanicus }\end{array}$ & 5 & & & & 5 & 5 & 5 & 5 & & & eed & & & 4 & $80 \%$ \\
\hline Fallugia paradoxa & & & & & & & & 1 & & $\begin{array}{ll}U \\
\sim S t\end{array}$ & $\begin{array}{l}\text { Inknowr } \\
\text { trugglin }\end{array}$ & & & 1 & $100 \%$ \\
\hline Salvia greggii & & 5 & & & 5 & 5 & 5 & 5 & & & & & 4 & mass & $n / a$ \\
\hline $\begin{array}{l}\text { Cytisus purgans 'Spanish } \\
\text { Gold' }\end{array}$ & 3 & & & & 1 & 1 & 1 & 1 & 0 & & & & & & $0 \%$ \\
\hline $\begin{array}{l}\text { Fendlera rupicola var. } \\
\text { wrightii }\end{array}$ & 1 & & & & 0 & & & & & & & & & & $0 \%$ \\
\hline
\end{tabular}

Figure 6. Woody shrub survival by year from 2007-2020. The plants categorized as 'survive' are listed alphabetically by genus at the top while those that are categorized as 'perish' are listed alphabetically by genus at the bottom.

\section{CONCLUSIONS}

Select plant taxa in each plant form category were successful on the green roof in this evaluation. Succulents showed greatest potential for survival on green roofs in a semi-arid climate. But the less studied shrub forms came in at a close second with only two taxa perishing. This evaluation demonstrates that all plant form categories can be utilized on a semiarid green roof, with (forms) being most successful. Forms such as upright forbs, creeping forbs, and graminoids, in general, were less successful. Therefore, taxa from these plant form types should be carefully selected for green roof use.

All plant forms demonstrate yearly fluctuation of relative success within taxa (i.e. individual plants have perished or survived), however relative plant cover on the green roof has remained constant over time. One of the most unexpected observations was $F$. paradoxa's spontaneous arrival on the green roof.

Seeding plants from all plant forms, such as E. nauseosa subsp. nauseosa var. nauseosa, E. leiomerus, and I. aggregata may increase success on a green roof due to vital seed dispersion and reappearance. Shade created by the installation of different plant heights such as woody shrubs may also increase the survivability of other plant forms, similar to how shade from solar 
panels improved green roof plant survivability in another Colorado study (Bousselot et al. 2017). Between low precipitation and low quantities of supplemental irrigation, this green roof demonstrates plant taxa that may be used on green roofs in other semi-arid climates.

In some cases, provenance may explain survivability in each of the plant form categories. In general, plants that are native to North America were more likely to survive on the green roof than plants that were not native; however, not equally or consistently across all plant categories. Factors such as low levels of supplemental irrigation, variable shading by taller plants, and microclimates could have greatly influenced the relative success or failure of species no matter their provenance. Additional long-term evaluations for both taxa survivability and provenance among green roofs in other regions of North America are needed.

\section{LITERATURE CITED}

Aguiar, A.C., S.A. Robinson and K. French. 2019. "Friends with benefits: The effects of vegetative shading on plant survival in a green roof environment." PLoS ONE, 14(11): 1-16. https://doi.org/10.1371/journal.pone.0225078

Bousselot, J.M., J.E. Klett and R.D. Koski. 2010. "Extensive green roof species evaluations using digital image analysis." HortScience, 45(8): 1288-1292. https://doi.org/10.21273/HORTSCI.45.8.1288

Bousselot, J.M., J.E. Klett and R.D. Koski. 2011. "Moisture content of extensive green roof substrate and growth response of 15 temperate plant species during dry down." HortScience, 46(3): 518522. https://doi.org/10.21273/HORTSCI.46.3.518

Bousselot, J., T. Slabe, J. Klett and R. Koski. 2017. "Photovoltaic array influences the growth of green roof plants." Journal of Living Architecture, 4(3): 9-18. https://doi.org/10.46534/jliv.2017.04.03.009

Braaker, S., M.K. Obrist, J. Ghazoul, M. Moretti and K. Cuddington. 2017. "Habitat connectivity and local conditions shape taxonomic and functional diversity of arthropods on green roofs." Journal of Animal Ecology, 86(3): 521-531. DOI: 10.1111/1365-2656.12648

Cao, J., S. Hu, Q. Dong, L. Liu, and Z. Wang. 2019. "Green roof cooling contributed by plant species with different photosynthetic strategies." Energy and Buildings, 195: 45-50. https://doi.org/10.1016/j.enbuild.2019.04.046

Chagolla-Aranda, M.A., E. Simá, J. Xamán, G. Álvarez, I. Hernández-Pérez, and E. Téllez-Velázquez. 2017. "Effect of irrigation on the experimental thermal performance of a green roof in a semiwarm climate in Mexico." Energy and Buildings, 154:232-243. https://doi.org/10.1016/j.enbuild.2017.08.082

CoCoRaHS. n.d. "Community Collaborative Rain, Hail and Snow Network." CoCoRaHS, www.cocorahs.org/.

Dunnett, N. and A. Nagase. 2010. "Drought tolerance in different vegetation types for extensive green roofs: effects of watering and diversity." Landscape and Urban Planning, 97(4): 318-327.

Dvorak, Bruce, and Astrid Volder. 2010. "Green Roof Vegetation for North American Ecoregions: A Literature Review." Landscape and Urban Planning, Elsevier. https://doi.org/10.1016/j.landurbplan.2010.04.009

Erwin, J. and J. Hensley. 2019. "Plants with horticultural and ecological attributes for green roofs in a cool, dry climate.” HortScience, 54(10): 1703-1711. https://doi.org/10.21273/HORTSCI13893$\underline{19}$

Getter, K.L. and D.B. Rowe. 2006. "The role of extensive green roofs in sustainable development." HortScience, 41(5): 1276-1285. 
Graceson, A., J. Monaghan, N. Hall, and M. Hare. 2014. "Plant growth responses to different growing media for green roofs." Ecological Engineering, 69: 196-200. https://doi.org/10.1016/j.ecoleng.2014.03.067

Kew Science. n.d. Plants of the World Online. http://www.plantsoftheworldonline.org/.

Klein, P.M. and R. Coffman. 2015. "Establishment and performance of an experimental green roof under extreme climatic conditions." Science of the Total Environment, 512: 82-93.

Köhler, M. and P. Poll. 2010. "Long-term performance of selected old Berlin greenroofs in comparison to younger extensive greenroofs in Berlin." Ecological Engineering 36: 722-729.

Kotsiris, G., P.A. Nektarios, N. Ntoulas and G. Kargas. 2013. "An adaptive approach to intensive green roofs in the Mediterranean climatic region." Urban Forestry and Urban Greening, 12(3): 380392. https://doi.org/10.1016/j.ufug.2013.05.001

Lubell, J.D., B. Connolly and K.N. Jones. 2017. "Ten-year persistence of native plant species on a green roof in Northeast :US." Native Plants Journal, 18(3): 227-234. https://www.muse.jhu.edu/article/680008.

Lundholm, J.T. and M. Cadotte. 2015. "Green roof plant species diversity improves ecosystem multifunctionality." Journal of Applied Ecology, 52(3): 726-734. https://doi.org/10.1111/1365$\underline{2664.12425}$

Lundholm, J., J.S. MacIvor, Z. MacDougall, and M. Ranalli. 2010. "Plant species and functional group combinations affect green roof ecosystem functions." PLOS ONE, 5(3): 1-11. https://doi.org/10.1371/journal.pone.0009677

Monterusso, M.A., D.B. Rowe, and C.L. Rugh. 2005. "Establishment and persistence of Sedum spp. and native taxa for green roof applications." HortScience, 40(2), 391-396.

Nektarios, P.A., I. Amountzias, I. Kokkinou and N. Ntoulas. 2011. "Green roof substrate type and depth affect the growth of the native species Dianthus fruticosus under reduced irrigation regimens." HortScience, 46(8): 1208-1216. https://doi.org/10.21273/HORTSCI.46.8.1208

Nektarios, P.A., I. Kokkinou and N. Ntoulas. 2020. "The effects of substrate depth and irrigation regime, on seeded Sedum species grown on urban extensive green roof systems under semi-arid Mediterranean climatic conditions." Journal of Environmental Management, p.111607.

Nektarios, P.A., N. Ntoulas, G. Kotopoulis, T. Ttoulou and P. Ilia. 2014. "Festuca arundinacea drought tolerance and evapotranspiration when grown on two extensive green roof substrate depths and under two irrigation regimes." European Journal of Horticultural Science, 79(3): 142-149.

Nektarios, P.A., N. Ntoulas, E. Nydrioti, I. Kokkinou, E.M. Bali and I. Amountzias. 2015. "Drought stress response of Sedum sediforme grown in extensive green roof systems with different substrate types and depths." Scientia Horticulturae, 181: 52-61. https://doi.org/10.1016/j.scienta.2014.10.047

Oberndorfer, E., J. Lundholm, B. Bass, R. Coffman, H. Doshi, N. Dunnett, S. Gaffin, M. Köhler, K. Liu, and B. Rowe. 2007. "Green roofs as urban ecosystems: ecological structures, functions, and services." Bioscience, 57(10): 823-833. doi:10.1641/b571005

Reyes, R., W. Bustamante, J. Gironás, P.A. Pastén, V. Rojas, F. Suárez, S. Vera, F. Victorero and C.A. Bonilla. (2016). "Effect of substrate depth and roof layers on green roof temperature and water requirements in a semi-arid climate." Ecological Engineering, 97: 624-632. https://doi.org/10.1016/j.ecoleng.2016.10.025

Schneider, A., M. Fusco, J. Bousselot. 2014. "Observations on the survival of 112 plant taxa on a green roof in a semi-arid climate." Journal of Living Architecture, 1(5): 10-30. https://doi.org/10.46534/jliv.2014.02.01.010

Shafique M., R. Kim, M. Rafiq. 2018. "Green roof benefits, opportunities and challenges - A review." Renewable and Sustainable Energy Reviews, 90: 757-773 https://doi.org/10.1016/j.rser.2018.04.006 
Thuring, C.E., R.D. Berghage and D.J. Beattie. 2010. "Green roof plant responses to different substrate types and depths under various drought conditions." HortTechnology, 20(2): 395-401. https://doi.org/10.21273/HORTTECH.20.2.395

Tran, S., J.T. Lundholm, M. Staniec, C.E. Robinson, C.C. Smart, J.A. Voogt and D.M. O'Carroll. 2019. "Plant survival and growth on extensive green roofs: A distributed experiment in three climate regions." Ecological Engineering, 127: 494-503. https://doi.org/10.1016/j.ecoleng.2018.09.027

Vahdati, N., A. Tehranifar and F. Kazemi. 2017. "Assessing chilling and drought tolerance of different plant genera on extensive green roofs in an arid climate region in Iran." Journal of

Environmental Management, 192: 215-223. DOI: 10.1016/j.jenvman.2017.01.027 